\title{
A Rede Brasileira de Monitoramento e Avaliação: um relato do processo de sua criação e desenvolvimento ${ }^{1}$
}

\author{
Afonso Henriques Borges² \\ Jânia Pinho ${ }^{3}$ \\ João Pedro Azevedo ${ }^{4}$ \\ John L. Newman ${ }^{5}$ \\ Juliana Wenceslau ${ }^{6}$ \\ Márcia Paterno Joppert ${ }^{7}$ \\ Sonia Nahas de Carvalho ${ }^{8}$
}

1 Os autores agradecem os comentários e sugestões dos demais membros da rede que participaram da V Conferência Internacional da Rede Latinoamericana de Monitoramento e Avaliação, em especial, Eder Campos e Lycia Lima. Os autores ainda gostariam de agradecer a iniciativa de Ricardo Santiago e o apoio de Ricardo Paes de Barros, Eduardo Rios-Neto e Pedro Olinto para o lançamento e realização do primeiro Seminário da Rede Brasileira de Monitoramento e Avaliação.

2 Ex-Presidente da Fundação João Pinheiro, atual Diretor Vice-Presidente do Escritório de Prioridades Estratégicas do Governo de Minas Gerais.

3 Gerente do Ambiente de Estudos, Pesquisas e Avaliação do Banco do Nordeste do Brasil.

4 Economista, Banco Mundial.

5 Economista Líder, Banco Mundial.

6 Doutoranda em Administração Pública, Programa de Pós-Graduação em Administração (PPGA), Universidade de Brasília (UnB).

7 Diretora-Geral da Agência Brasileira de Avaliação.

8 Assessora Técnica da Diretoria Adjunta de Análise e Disseminação de Informações (Fundação Seade). 


\section{Resumo}

O PRESENTE ARTIGO TEM COMO OBJETIVO APRESENTAR O HISTÓRICO DE CRIAÇÃo DA REDE BRASILEIRA DE MONITORAMENTO E AVALIAÇÃO, ASSIM COMO ALGUNS DOS SEUS AVANÇOS RECENTES E DESAFIOS FUTUROS. DENTRE OS PRINCIPAIS AVANÇOS, DESTACAM-SE: A FILIAÇÃO DE MAIS DE 1.500 MEMBROS NOS SEUS 23 MESES DE VIDA E AS MAIS DE 60.000 VISITAS AO SEU SITE 9 . AlGUNS dOS IMPORTANTES DESAFIOS FUTUROS SÃO A NECESSIDADE CONTÍNUA DE ATRAIR E RETER SEUS MEMBROS, A CAPACITAÇÃO DOS SEUS MEMBROS NO USO DA

PLATAFORMA WEB 2.0 E A CAPACIDADE DA REDE DE RENOVAR SUAS LIDERANÇAS.

\begin{abstract}
THIS ARTICLE PRESENTS THE HISTORY OF CREATION OF THE BRAZILIAN MONITORING AND EVALUATION NETWORK AS WELL AS SOME OF ITS RECENT ADVANCES AND FUTURE CHALLENGES. AMONG SEVERAL IMPROVEMENTS, THERE ARE: THE AFFILIATION OF MORE THAN 1.500 MEMBERS IN ITS 23 MONTHS OF EXISTENCE AND THE RECORD OF MORE THAN 60.000 MONTHLY VISITS TO THE SITE. SOME OF THE IMPORTANT CHALLENGES OF THE FUTURE ARE THE ONGOING NEED TO ATTRACT AND RETAIN MEMBERS, THE NECESSITY TO TRAIN ITS MEMBERS IN THE USE OF WEB 2.0 PLATFORM AND THE NETWORK CAPACITY TO RENEW ITS LEADERSHIP.
\end{abstract}

\section{PALAVRAS-CHAVE:}

Programas sociais; Avaliação; Monitoramento; Histórico

Revista Brasileira de Monitoramento e Avaliação | Número 1 | Janeiro-Junho de 2011

A Rede Brasileira de Monitoramento e Avaliação: um relato do processo de sua criação e desenvolvimento 


\section{Antecedentes}

O processo de valorização da gestão pública no Brasil teve início na década de 90. O Plano Diretor da Reforma do Aparelho do Estado, publicado em setembro de 1995, coloca a gestão como um instrumento indispensável para consolidar a estabilização econômica e assegurar o crescimento sustentado da economia. Reforça os conceitos e a necessidade da formação de um Estado mais eficiente.

Logo em seguida e juntamente com o processo de controle inflacionário e de estabilidade econômica, que permitiu ao mercado mais bem planejar seus investimentos e seus projetos de futuro, começa a ter expressão no Brasil a gestão para resultados. Este movimento ocasionou forte impacto no Governo Federal brasileiro, influenciando a atuação das áreas centrais e setoriais, o controle interno e externo, além das atividades dos organismos internacionais e da sociedade civil. A adoção destes novos procedimentos de gestão pública ancora-se nos preceitos constitucionais de 1988 (artigo 165) de instituição das normas de regulamentação do processo orçamentário federal, com a introdução, de forma integrada, de três instrumentos: Plano Plurianual de Investimentos (PPA), Lei de Diretrizes Orçamentárias (LDO) e Lei Orçamentária Anual (LOA). As constituições estaduais e as leis orgânicas municipais reproduziram os preceitos da Constituição Federal. Como recurso a novas formas de gestão pública, o PPA veio representar a aproximação entre planejamento e gestão orçamentária em pelo menos três direções ao: (i) implementar o planejamento governamental no médio e longo prazos; (ii) coordenar ações governamentais e (iii) estabelecer diretrizes, objetivos e metas da administração pública que orientassem a alocação dos recursos públicos.

$\mathrm{Na}$ área central, o principal ator deste processo tem sido o Ministério do Planejamento, Orçamento e Gestão (MPOG). Nos últimos dez anos, o MPOG tem realizado um esforço de incorporar o enfoque de resultados nas políticas públicas setoriais. Tal esforço foi iniciado com o PPA 2000-2003, por meio de três grandes inovações: a primeira foi basear os investimentos em estudos de planejamento territorial, o que resultou em um portfólio composto por 952 projetos cujo orçamento totalizava R\$ 317 bilhões. Classificado em macro setores ${ }^{10}$, considerava a integração entre as ações, a responsabilização pelos resultados e o aporte de recursos pelo mercado privado. A segunda foi incluir indicadores de implementação e resultado, o que promoveu o início efetivo de uma cultura de gestão para resultados e de conceitos de monitoramento e avaliação (M\&A). A terceira foi alocar um gerente responsável por programa, por meio da prática de responsabilização para resultados. Com isso, buscavase promover uma cultura empreendedora no Governo Federal, introduzindo ferramentas de gerenciamento de programas e projetos e capacitando os gestores públicos para utilizá-las. Entre os avanços recentes do MPOG, destacase a publicação do guia metodológico para indicadores de programas (BRASIL, 2010).

Os Ministérios setoriais também iniciaram um esforço de monitorar e avaliar suas políticas, especialmente as áreas de Educação, Saúde e Desenvolvimento Social. Criado em 2003, 
o Ministério do Desenvolvimento Social e Combate à Fome (MDS) tem se destacado por ser o primeiro a institucionalizar uma secretaria especializada em avaliação e gestão da informação, a qual tem contratado estudos, promovido treinamentos e implementado sistemáticas para o monitoramento de políticas de transferência de renda, assistência social e segurança alimentar (VAITSMAN et al., 2006). A Secretaria de Avaliação e Gestão da Informação (SAGI) é responsável por grande volume de estudos, pesquisas de linha de base, surveys de beneficiários e avaliações de impacto, produzidos para subsidiar as políticas sociais (WENCESLAU; HOBBS, 2008).

O controle interno do Governo Federal, por sua vez, passou por um processo de fortalecimento institucional a partir de 2002, por meio da criação da Controladoria-Geral da União (CGU). A CGU é resultado da fusão entre a Secretaria Federal de Controle Interno (SFC) e a Comissão de Coordenação de Controle Interno (CCCI), alcançando o status de Ministério. A atuação da CGU tem estimulado a transparência e qualidade das informações públicas, focalizando a prevenção, fiscalização e controle da aplicação de recursos federais. Utiliza quatro instrumentos: fiscalização de municípios por sorteio, auditorias financeiras anuais, investigações de casos e Balanço Geral da União. Ferraz e Finan (2008) demonstram que o eleitor brasileiro, quando bem informado, rejeita os políticos acusados de corrupção e prefere os candidatos honestos. A divulgação das auditorias da CGU nos municípios onde foram detectados muitos casos de corrupção reduziu em $20 \%$ as chances de reeleição dos prefeitos.

O Poder Legislativo, por meio do Tribunal de Contas da União (TCU), passou a aferir o desempenho das políticas governamentais para realizar o controle externo, além do tradicional enfoque em aspectos de conformidade e legalidade. A partir de 1999, o TCU passou a realizar auditorias de natureza operacional, incorporando técnicas como estudo de caso, surveys e grupos focais, utilizadas em avaliações de implementação de programas. A inclusão da performance audit entre as auditorias realizadas pelo TCU ocorreu a partir da assistência técnica promovida pelo UK Department of International Development (DFID) e troca de experiências com o National Audit Office (NAO), ambos do Reino Unido, e com o Government Accountability Office (GAO) dos Estados Unidos (FLINT, 2004).

Nos últimos anos, alguns estados e municípios também têm demonstrado um crescente interesse pela agenda de monitoramento e avaliação. Dentre estes, cabe destacar Minas Gerais, que, por meio da Secretaria de Planejamento, de seu Programa Gestão por Resultado e da Fundação João Pinheiro, vem 
desenvolvendo e promovendo um conjunto de práticas inovadoras na gestão pública brasileira, como uma forte ênfase no uso de indicadores e metas para dar foco e direção à administração pública (SEPLAG/MG, 2008; SEPLAG/MG, 2009).

Dentre as instituições estaduais existentes no Brasil, destaca-se também o trabalho da Fundação Seade ${ }^{11}$, que vem especializandose na última década em criar ou aprimorar metodologias e ferramentas para formulação, monitoramento e avaliação de políticas e programas públicos, particularmente na área social. As atividades desenvolvidas pela instituição nesse campo fornecem insumos para as diferentes fases da gestão pública, auxiliando o desenho de programas e políticas, facilitando eventuais ajustes e correções de rumos. Em apoio a agências executoras ou de coordenação das ações de governo, essas atividades compreendem a realização de estudos de avaliação de programas públicos específicos, a construção de metodologias de monitoramento, com definição de indicadores, para implementar o acompanhamento de políticas ou agências públicas e, com objetivo semelhante, a estruturação de amplos sistemas de informação gerencial.

Outro grupo de instituições que também atua no campo de monitoramento e avaliação no Brasil são os bancos de desenvolvimento, com um destaque particular para o Banco do Nordeste do Brasil ${ }^{12}$ (BNB). Entre algumas das iniciativas de destaque encontram-se a avaliação do Programa Crediamigo, realizada em parceria com o Banco Mundial (BM), e a avaliação do Fundo Constitucional de Financiamento do Nordeste (FNE). Este último programa conta com um sistema de avaliação que utilizou dados primários e secundários. Foram avaliados os subprogramas FNE-Agroindustrial, o FNE-Comércio-Serviços e Industrial, o FNE-Rural e avaliações globais do Fundo.

O setor privado brasileiro também desempenha um papel importante no fortalecimento desta agenda no Brasil. Iniciativas como o Movimento Brasil Competitivo (MBC), criado em novembro de 2001, têm como objetivo principal viabilizar projetos que visam ao aumento da competitividade das organizações e da qualidade de vida da população, inclusive no modelo de gestão do setor público. Esta iniciativa já atendeu vários Ministérios, estados e municípios no Brasil, sempre com o objetivo de promover a adoção de práticas de gestão baseada em resultados.

Várias organizações e movimentos da sociedade civil têm também atuado no monitoramento e avaliação de programas, na contratação de estudos, na organização de seminários e na demanda por informação, tais como a Fundação Itaú Social, o Instituto Unibanco e o Canal Futura.

Por fim, como exemplo de iniciativas de organismos internacionais, há o Programa Brasil Avaliação (BRAVA), desenvolvido pelo Banco Mundial como um projeto de assistência técnica, não vinculado a operações de empréstimo, com o intuito de auxiliar o Governo Federal no desenvolvimento de sistemas e atividades de M\&A. O BRAVA foi lançado em 2005 e finalizado em 2009, tendo como principal parceiro a Câmara Técnica de Monitoramento e Avaliação (CTMA), um 
comitê composto pelos principais órgãos do Governo Federal interessados no assunto. Os objetivos do BRAVA eram: (a) auxiliar no desenvolvimento de metodologias e da política de M\&A para o Governo Federal e seus Ministérios, (b) disseminar a cultura de M\&A e (c) preparar sistemas para Gestão para Resultados (GpR) com o intuito de: (i) esclarecer os papéis institucionais na coordenação e implementação da GpR, (ii) estabelecer os principais incentivos para que Ministérios fizessem M\&A de seus programas, (iii) desenvolver padrões de qualidade para M\&A e (iv) promover a utilização e disseminação das informações geradas pelo M\&A ${ }^{13}$.

\section{A Rede Brasileira de Monitoramento e Avaliação}

O processo de redemocratização brasileiro fortalece cada vez mais o papel da sociedade, especialmente como agente direto do processo de formulação, acompanhamento e controle da implementação de políticas públicas. A participação no processo decisório e o controle que a sociedade atualmente exerce sobre os atos dos governantes e de seus representantes eleitos - em fóruns e conselhos constituídos especificamente para este fim - têm fornecido maior legitimidade às políticas públicas. Além disso, é importante destacar as novas formas de participação do setor privado na implantação de políticas, como as parcerias público-privadas, consórcios público-privados que também induzem uma importância crescente à avaliação e aos instrumentos disponíveis para realizá-la.

No Brasil, o conhecimento e a prática na área de monitoramento de programas e projetos são vastos, como visto na seção anterior. Entretanto, a maior parte das experiências realizadas está armazenada em centros de pesquisa e ensino ou no acervo pessoal de consultores. Portanto, o acesso depende da "garimpagem" por parte dos interessados pelo tema e da capacidade de disseminação dos que produziram determinado conhecimento. Assim, o conhecimento existente sobre avaliação de programas e políticas no Brasil é ainda disperso e não sistematizado. A partir desta situação, surgiu a ideia, entre os especialistas no tema, de se promover uma iniciativa que viesse a preencher essa lacuna e que fosse capaz de alcançar e sen-

\footnotetext{
11 http://www.seade.gov.br/

12 http://www.bnb.gov.br/
}

\begin{abstract}
13 Em seus cinco anos de existência, o BRAVA prestou assistência, fez estudos e promoveu eventos em conjunto com o Governo Federal, estados e municípios. Dentre eles, destacam-se: (i) workshops sobre sistemas de avaliação do Chile, da Austrália e do Canadá; (ii) elaboração de metodologia para revisar o marco lógico dos programas do Plano Plurianual (PPA); (iii) avaliação institucional do sistema de avaliação do PPA e do MDS; (iv) participação no desenho de avaliações de programas, como "Bolsa Família", "Brasil Alfabetizado", "Crediamigo", "Quilombolas", "Saúde Indígena"; (v) capacitação de auditores do TCU em avaliação de impacto, por meio da doação IDF, e (vi) auxílio ao estado de Minas Gerais no desenho da primeira Pesquisa por Amostra de Domicílios (PAD-MG), metodologia das avaliações de impacto e benchmarking de indicadores.
\end{abstract}

Revista Brasileira de Monitoramento e Avaliação | Número 1 | Janeiro-Junho de 2011 
sibilizar as instituições que acumulam experiências na área, realizando uma melhor gestão do seu conhecimento.

As redes de organizações buscam formar um ambiente de discussões entre entidades afins e o fortalecimento destas pela troca de informações, promoção de congressos, apoio jurídico, desenvolvimento e disponibilização de base de dados, relacionada aos interesses das instituições participantes. A organização, o registro e a socialização de informações, por parte de seus elementos constituintes, podem criar um sistema mais propenso à inovação e à interação com o ambiente externo. A disseminação das informações organizadas e estruturadas para o ambiente externo possibilita maior visibilidade e abertura para agregação de elementos novos, com vistas a reconfigurações e aprimoramento do sistema atual (LOPES, 1996).

Ogerenciamento de informaçõesmostrou-se, indiscutivelmente, um fator essencial nas redes, tanto no que se refere à busca de novos elos, criando maiores possibilidades de atuar no contexto dinâmico da realidade de que faz parte e garantindo sua manutenção e sobrevivência, quanto na criação de mecanismos de controle interno que promovam o melhor aproveitamento de seus recursos. A seguir, serão explicados o processo de formação, objetivos, arranjo institucional, ferramenta tecnológica e resultados dos dois primeiros anos da Rede Brasileira de Monitoramento e Avaliação.
3. O processo de formação da Rede Brasileira de Monitoramento e Avaliação

\subsection{OBJETIVOS E ESTRATÉGIAS}

A Rede Brasileira de Monitoramento e Avaliação surgiu como um capítulo da Rede de Monitoramento e Avaliação da América Latina e Caribe ${ }^{14}$, tendo como princípios fundadores: (i) a descentralização, (ii) a abertura e (iii) o baixo custo de fracasso. Nestes dois primeiros anos de vida, a iniciativa do capítulo brasileiro caracterizou-se por não ter apenas um único champion institucional. Ao contrário, diversas instituições, como as fundações estaduais de estatística e economia, bancos públicos e organismos internacionais, apoiam esta iniciativa. Em geral, os representantes dessas instituições na rede, além de pontos focais, representam indivíduos comprometidos com este tema em suas respectivas instituições. Um segundo princípio importante da rede é a sua abertura, traduzida pela total liberdade de filiação, seja pessoal, institucional, ou de outras redes, buscando com isso maximizar as possibilidades de colaboração entre os diferentes atores interessados neste tema no Brasil. Por último, um terceiro e importante princípio foi a busca por uma configuração que diminuísse ao máximo as perdas em caso de fracasso da iniciativa. Ou seja, a existência desta rede só se justifica se ela for útil e capaz de demonstrar o seu valor, o que tem-se dado pelas crescentes filiações, acessos, interações e contribuições. 
A Rede Brasileira de Monitoramento e Avaliação foi assim lançada em dezembro de 2008 sob a liderança da Fundação João Pinheiro (FJP), do Banco Interamericano de Desenvolvimento (BID) e do Banco Mundial (BM). A motivação foi o reconhecimento da existência de uma massa crítica considerável sobre este tema no Brasil, a ausência de espaços de troca e sistematização de experiências e o efeito mobilizador da $4^{\mathrm{a}}$ Conferência Internacional da Rede Regional, sediada em Belo Horizonte, em 2008. A proposta, portanto, foi utilizar a concentração de diversos atores envolvidos neste tema no Brasil, durante os dias da Conferência, para mobilizar um evento paralelo e propor a fundação do capítulo brasileiro da Rede da América Latina e Caribe.

Além da FJP, do BM e do BID, participaram da reunião de fundação diversos representantes de instituições internacionais e nacionais, como: o escritório brasileiro do Programa das Nações Unidas para o Desenvolvimento (PNUD), a Fundação Seade, o Banco do Nordeste do Brasil, a Fundação Itaú Social, o Instituto Unibanco, o Ministério do Desenvolvimento Social e Combate à Fome (MDS), o Tribunal de Contas da União (TCU), a Secretaria de Planejamento do Estado do Ceará, entre outros.

Como resultado das discussões, foram definidos os objetivos fundamentais da rede, quais sejam:
Promover o intercâmbio de experiências e conhecimento e disseminar informações relativas a M\&A, por meio da organização de seminário anual e da utilização de uma plataforma de rede social, tendo por missão fortalecer a cultura de monitoramento e avaliação no Brasil.

Seu público-alvo são atores públicos e privados com atuação no campo de M\&A de organizações, políticas, programas e projetos, incluindo: gestores, pesquisadores, consultores, professores, estudantes, estudiosos e organizações financiadoras, promotoras e avaliadoras, bem como outras redes afins.

\subsection{O ARRANJO INSTITUCIONAL}

Em junho de 2009, durante o I Seminário Nacional da Rede, em Belo Horizonte, foi realizada uma segunda reunião, que contou com a participação de representantes das seguintes instituições: Banco Mundial; Fundação João Pinheiro; Governos de São Paulo (Fundação Seade), Ceará (Seplag), Rio Grande do Sul (Secretaria de Planejamento e Gestão, Secretaria da Fazenda e Fundação de Economia e Estatística), Bahia (Seplag), Espírito Santo; Banco do Nordeste do Brasil; TCU; PNUD; Ministério do Desenvolvimento Agrário (Secretaria de Desenvolvimento Territorial); MDS (Secretaria de Avaliação e Gestão da Informação); Ministério da Educação (Secretaria de Educação Continuada, Alfabetização e Diversidade); Fundação Itaú Social; Instituto Ecofuturo e Instituto Unibanco; Agência Brasileira de Avaliação;

14 Iniciativa promovida pelo Banco Mundial, Banco Interamericano de Desenvolvimento e Governo da Espanha.

Revista Brasileira de Monitoramento e Avaliação | Número 1 | Janeiro-Junho de 2011 
Herkenhoff Prates - Instituto de Tecnologia e Desenvolvimento de Minas Gerais; Instituto Ayrton Senna; Universidade Federal de Viçosa; Cecaps e A \& M Consultoria.

Nesta reunião, foram tomadas algumas decisões em relação ao arranjo institucional, sendo as principais:

- definição de um Comitê Gestor, formado por representantes da FJP, BM, BNB, Fundação Seade e Agência Brasileira de Avaliação;

- definição dos tipos de membros: membros institucionais e membros pessoas físicas, além de outras redes.

Foi reiterada ainda a necessidade de se dispor de uma ferramenta tecnológica materializada numa página de internet, de forma a publicar os conteúdos produzidos pela rede e outras informações.

\subsection{SEMINÁRIOS NACIONAIS}

Um dos objetivos iniciais da rede era a organização de um evento anual no qual as partes interessadas poderiam expor seus trabalhos, trocar experiências e identificar melhores práticas no campo do monitoramento e avaliação de políticas públicas.

No primeiro seminário, optou-se por definir a programação do evento enfocando os diferentes tipos de avaliação no ciclo das políticas públicas, tais como: diagnósticos para o desenho de políticas públicas, marco lógico, avaliações ex-ante, avaliações de implementação e avaliações ex-post. Outro princípio organizador das seções foi a maior ênfase no como implementar e utilizar estas ferramentas e menor nos resultados gerados por estes processos.

Para o segundo seminário, o princípio organizador foi mantido, com o acréscimo de alguns temas que despertam mais interesse entre gestores públicos, como seleção de indicadores e metas para acordos de gestão.

\subsection{PLATAFORMA TECNOLÓGICA}

A página da rede na internet foi concebida a partir dos conceitos de internet 2.0 e suportada por uma solução tecnológica denominada "Ning". Esta plataforma de comunidades digitais permite criar redes sociais e o seu uso resultou na página cujo endereço é: <http:// redebrasileirademea.ning.com>, criada em junho de 2009, logo após a segunda reunião.

As principais funcionalidades da plataforma selecionada são:

- possibilidade de publicar notícias por meio de um blog;

- possibilidade de criar fóruns de discussão;

- possibilidade de criar grupos temáticos;

- possibilidade de publicar um calendário de eventos;

- criação de um perfil individual dos membros;

- possibilidade de anexar arquivos;

— interface amigável (de fácil operação);

- visualmente atrativo. 
- Figura 1: PÁGinA dA PLATAFORMA WEB 2.0

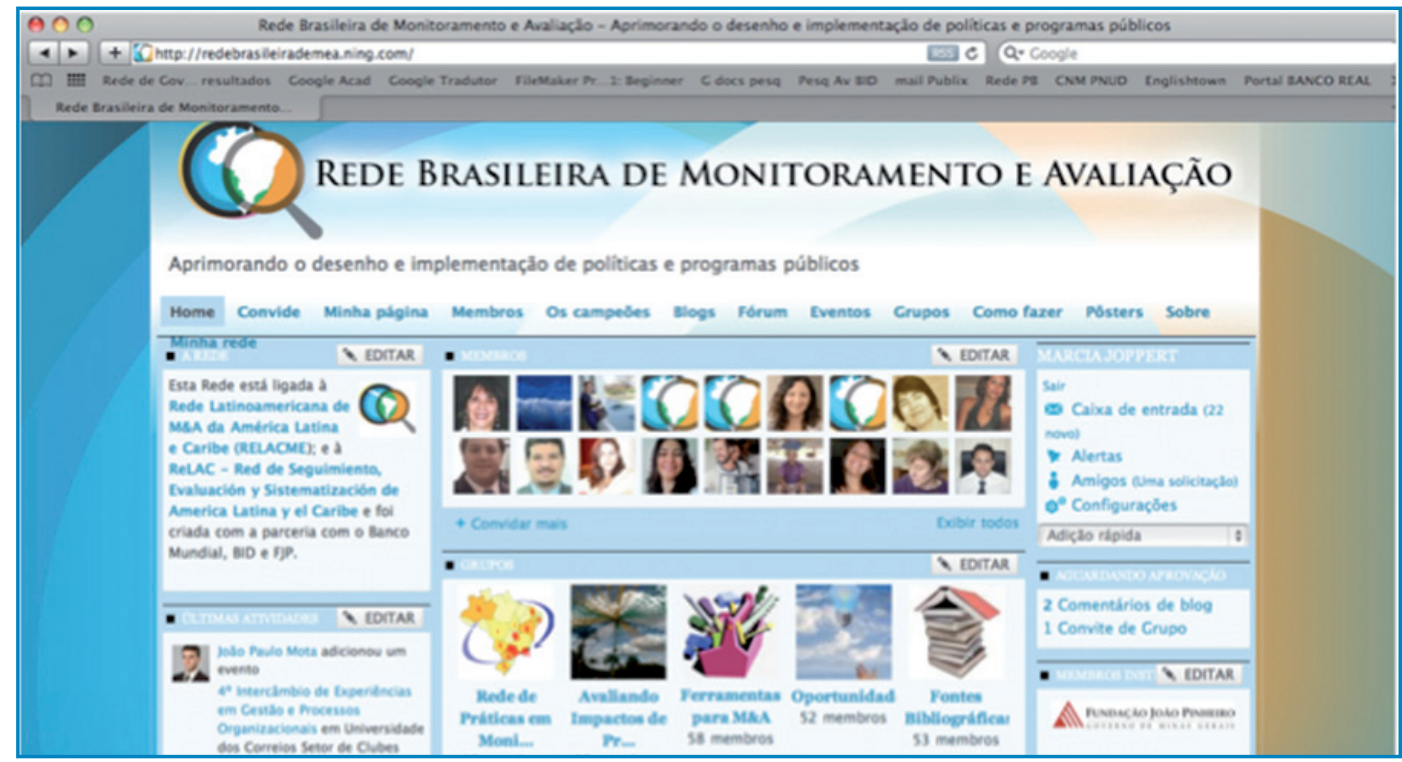

Fonte: http://redebrasileirademea.ning.com/

Um aspecto fundamental de iniciativas desta natureza é o seu planejamento, em particular no que diz respeito à estratégia de lançamento, divulgação e comunicação da iniciativa. É de fundamental importância considerar alguns aspectos básicos, como: (i) antes de lançar sua página, procure alimentá-la com conteúdos relevantes para a população-alvo (ninguém gosta de se tornar membro de uma cidade fantasma); (ii) inicie o seu site com um número pequeno de funcionalidades (i.e. blog, vídeos e eventos) e acrescente outras à medida que o tráfego aumentar (i.e. fóruns, grupos); (iii) tire proveito de eventos presenciais para divulgar sua rede ${ }^{15}$.
A evolução do gerenciamento desta plataforma também é algo interessante. Quando foi lançada, optou-se por uma página com uma estrutura relativamente fechada, com poucos administradores e com conteúdo disponível apenas para os membros. Gradualmente, a rede ganhou agilidade, abertura e removeu muitas destas restrições que, na prática, mostravam-se burocráticas. Atualmente a rede conta com quatorze. A mudança mais recente foi a total abertura do conteúdo da rede, tornando a filiação apenas necessária para a postagem de mensagens e eventos assim como para a participação em grupos temáticos e fóruns de discussão.

15 http://help.ning.com/cgi-bin/ning.cfg/php/enduser/workshop.php?p_sid=LDXLDYWj

Revista Brasileira de Monitoramento e Avaliação | Número 1 | Janeiro-Junho de 2011 


\section{Resultados}

Um aspecto estratégico para a consolidação de redes é o monitoramento dos seus resultados - por meio da construção e atualização de um perfil dos seus membros - e do tráfego de visitas na sua página na web.

\subsection{MEMBROS}

A Rede Brasileira de M\&A conta atualmente com mais de 1.500 membros $^{16}$ dos 27 estados do Brasil e de 26 países. A figura 2 mostra a evolução do número de membros da rede desde seu lançamento. As descontinuidades observadas estão claramente associadas a eventos específicos durante os quais membros da rede, direta ou indiretamente envolvidos na organização destes, aproveitaram a oportunidade para divulgá-la. E o resultado, em termos de filiação, é notável.
Os membros da rede são na sua maioria profissionais e instituições públicas e privadas envolvidas em atividades de monitoramento e avaliação ${ }^{17}$. Algumas das características principais da rede são:

- as mulheres predominam com $60 \%$ das filiações;

- $\quad 10 \%$ dos membros têm entre 17 e 25 anos de idade; $82 \%$, entre 26 e 55 anos e $8 \%$ com mais 55 anos de idade;

- o membro mais velho possui 77 anos de idade;

- em termos de ocupação, 48\% são servidores públicos, 14\% são da área educacional, $12 \%$ são de ONGs, 10\% são do mercado privado, $5 \%$ são estudantes, 3\% são consultores e 3\% são oriundos

- FIGURA 2: EVOLUÇÃO DAS INSCRIÇÕES NA REDE DESDE SEU LANÇAMENTO

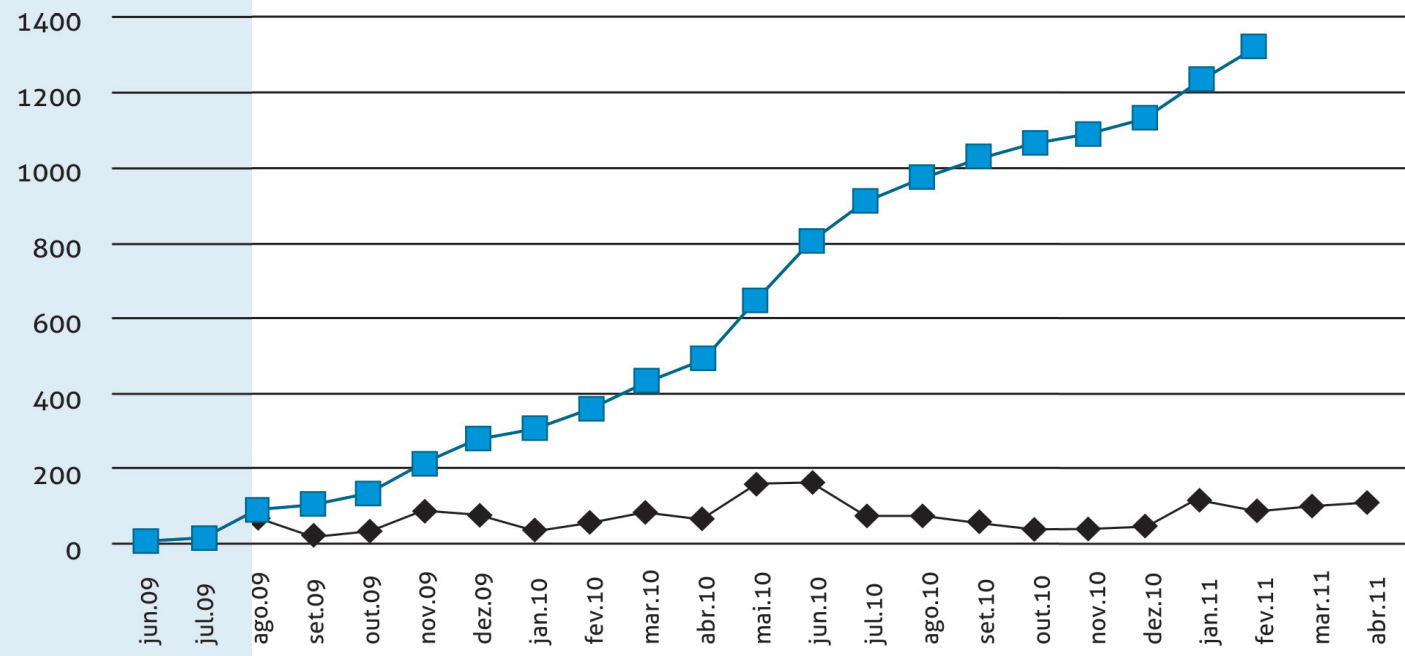


de núcleos de estudos e pesquisas. Os demais participantes (menos de 3\% cada) são oriundos de organismos e bancos internacionais, bancos públicos nacionais e associações de classe/empresariais;

- 55\% dos membros conheceram a rede por meio de um colega e outros $13 \%$ por meio de algum evento onde a rede foi divulgada.

\subsection{MONITORAMENTO DOS ACESSOS À PÁGINA}

Outro aspecto importante da rede é o monitoramento do seu uso. Atualmente, muitas ferra- mentas gratuitas encontram-se à disposição para facilitar este propósito. Essas ferramentas permitem o acompanhamento em tempo real do desempenho da rede, coletam diversas estatísticas de uso, incluindo o número de visitas, países, cidades e sites de origem. Além disso, é possível construir dashboards, como o apresentado na figura 3, que, entre outras coisas, permitem a comparação da performance do tráfego da rede ao longo do tempo e de sítios da mesma natureza, além do envio automático de relatórios para seus membros. Esse tipo de monitoramento permite informar o comitê executivo da rede sobre o seu desempenho e melhor planejar as ações de mobilização na iniciativa.

\section{- FIGURA 3. DASHBOARD DA REDE BRASILEIRA DE MONITORAMENTO E AVALIAÇÃO}

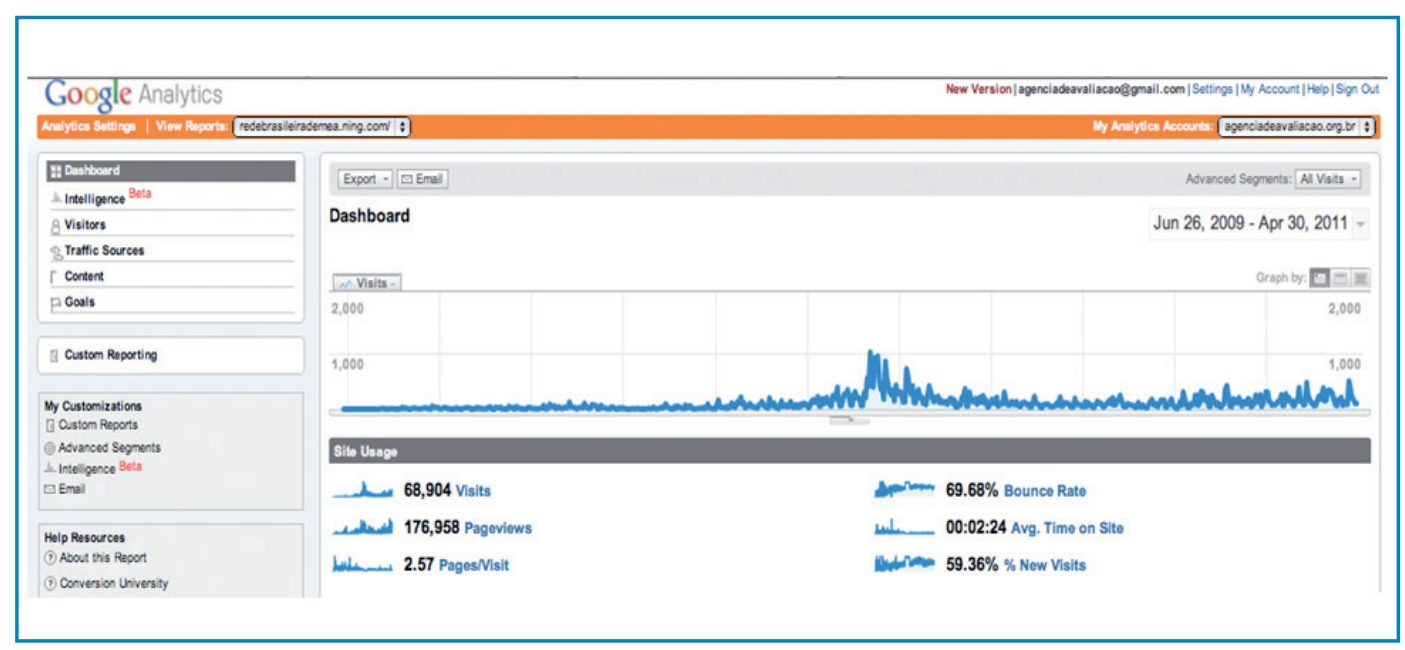

16 Dados referentes a 30 de abril de 2011.

17 Um perfil detalhado dos membros pode ser encontrado na página da rede.

Revista Brasileira de Monitoramento e Avaliação | Número 1 | Janeiro-Junho de 2011 


\subsection{PUBLICAÇÃO DE CONTEÚDOS}

Outra métrica interessante diz respeito ao nível de atividade da publicação de conteúdos na plataforma. Até o momento, já foram postados mais de 260 blogs, por volta de 100 eventos e 40 referências bibliográficas comentadas pelos membros da rede. Além disso, o número de comentários entre os participantes também tem aumentado, sugerindo um maior engajamento dos membros.

\section{Considerações finais e recomendações}

A Rede Brasileira de Monitoramento e Avaliação configura-se numa parceria de diversas organizações de distintas naturezas. Dentre alguns dos seus indicadores de sucesso, destaca-se a capacidade de atrair e reter membros: apenas 17 pessoas cancelaram suas inscrições como membros. No entanto, no que se refere aos seus principais desafios, merece atenção a construção de sua sustentabilidade. Esta sustentabilidade será função da capacidade da rede de manter a apresentação de conteúdos que sejam relevantes para seus membros, a alternância de lideranças no seu comitê executivo e a atração de novos facilitadores da rede, em particular para os grupos temáticos. Outro desafio importante é a desconcentração geográfica dos membros e conteúdos apresentados, tornando o espaço da rede de utilidade para membros e instituições nos mais distintos estágios de experiência. Uma última preocupação é a manutenção e ampliação da abertura da rede, evitando a percepção que este possa ser um espaço capturado por um pequeno grupo de instituições.
Do ponto de vista da plataforma web implementada, algumas dificuldades merecem destaque: (i) o uso inadequado dos "espaços virtuais", tendo em vista que muitos membros têm tido dificuldade para entender as diferenças entre blogs, fóruns, grupos temáticos e campos de comentários; com isso, ignoram as diferenças conceituais entre estes e postam seus conteúdos de maneira indiscriminada; (ii) outro ponto observado é a postagem de conteúdos (blogs e comentários) sem links para as referências sobre o tema e (iii) a dificuldade que muitos membros possuem com a apresentação visual dos seus blogs, ignorando muitas das funções de edições disponíveis pela ferramenta e, como consequência, o layout da página principal fica muitas vezes confuso.

Algumas das lições para outras redes desta natureza são: (i) a necessidade da existência de uma massa crítica de conteúdos e entidades que produza e demande conhecimento sobre este tema é de fundamental importância; (ii) o caráter de pluralidade institucional, como objetivo explícito de blindar a iniciativa dos naturais ciclos políticos e/ou de um número reduzido de champions; (iii) é fundamental contar e formar um grupo de animadores que auxiliem na produção de conteúdos na página da rede. Dentre as diversas funções destes animadores, destacam-se: selecionar e angariar membros; conhecer e analisar o perfil dos membros (pessoas e instituições); alimentar o site com conteúdos de interesse e que atendam as expectativas dos membros; promover a comunicação entre os membros; elaborar relatórios periódicos de atividades; conectar-se a iniciativas correlatas; divulgar a iniciativa e articular-se. 


\section{Referências bibliográficas}

BRASIL. Ministério do Planejamento, Orçamento e Gestão. Indicadores de programas: guia metodológico. Brasília, DF: Secretaria de Planejamento e Investimentos Estratégicos, 2010. 128 p.

CADERNOS de indicadores 2009: indicadores da gestão por resultados do governo do Estado de Minas Gerais. Belo Horizonte, MG: SEPLAG, 2009.

FERRAZ, Claudio; FINAN, Frederico. Exposing corrupt politicians: the effects of Brazil's publicly released audits on electoral outcomes. The Quarterly Journal of Economics. MIT Press, v. 123, n. 2, pag.703-745, 2008.

FLINT, M. Country study: Brazil 1997-2003. In: EVALUATION of DFID Country Programmes. DFID, 2004.
LOPES, Sônia Aguiar. A teia invisível - informação e contra-informação nas redes de ongs $e$ movimentos sociais. Rio de Janeiro: Universidade Federal do Rio de Janeiro, 1996. Tese de Doutorado.

NOTA Técnica n. 37 /2008. Belo Horizonte: DCMG/SEPLAG, 2008.

VAITSMAN, Jeni; RODRIGUES, Roberto W. S.; PAES-SOUSA, Rômulo. The system for evaluating and monitoring social development programs and policies: the case of the Ministry of Social Development and the Fight Against Hunger in Brazil. Brasília: MDS; UNESCO, 2006. Policy papers17.

WENCESLAU, J.; HOBBS, J. Institutional assessment of monitoring and evaluation systems (M\&E) in the Ministry of Social Development and Combating Hunger (MDS).World Bank, 2008. 